\title{
Weak convergence for the minimal position in a branching random walk: a simple proof
}

\author{
by \\ Elie Aïdékon and Zhan Shi \\ Technische Universiteit Eindhoven \& Université Paris VI \\ Dedicated to Professors Endre Csáki and Pál Révész \\ on the occasion of their 75th birthdays
}

\begin{abstract}
Summary. Consider the boundary case in a one-dimensional super-critical branching random walk. It is known that upon the survival of the system, the minimal position after $n$ steps behaves in probability like $\frac{3}{2} \log n$ when $n \rightarrow \infty$. We give a simple and self-contained proof of this result, based exclusively on elementary properties of sums of i.i.d. real-valued random variables.
\end{abstract}

Keywords. Branching random walk, minimal position.

2010 Mathematics Subject Classification. 60J80.

\section{Introduction}

Consider a (discrete-time, one-dimensional) branching random walk. It starts with an initial ancestor particle located at the origin. At time 1, the particle dies, producing a certain number of new particles; these new particles are positioned according to the law of a given finite point process. At time 2, these particles die, each giving birth to new particles that are positioned (with respect to the birth place) according to the law of the same point process. And the system goes on indefinitely, as long as there are particles that are alive. We assume that, each particle produces new particles independently of other particles in the same generation, and of everything up to that generation. 
The number of particles in each generation obviously forms a Galton-Watson process, which will always be assumed to be super-critical.

Let $(V(x),|x|=n)$ be the positions of the particles at the $n$-th generation. The process $(V(x))$ indexed by a Galton-Watson tree is called a branching random walk. We do not assume the random variables $V(x),|x|=1$, to be independent, nor necessarily identically distributed, though it is often assumed in the literature (for example, in [16]).

We are interested in $\min _{|x|=n} V(x)$, the minimal position of the branching random walk after $n$ steps. Under a mild integrability assumption, we have (Kingman [11], Hammersley [7], Biggins [2]), on the set of non-extinction,

$$
\frac{1}{n} \min _{|x|=n} V(x) \rightarrow \gamma, \quad \text { a.s. }
$$

where $\gamma \in \mathbb{R}$ is a known constant.

The rate of convergence in (1.1) has recently been studied, independently, by $\mathrm{Hu}$ and Shi [8], and Addario-Berry and Reed [1]. To state the result, we assume the following condition:

$$
\mathbf{E}\left(\sum_{|x|=1} \mathrm{e}^{-V(x)}\right)=1, \quad \mathbf{E}\left(\sum_{|x|=1} V(x) \mathrm{e}^{-V(x)}\right)=0 .
$$

This is referred to in the literature as the boundary case; see for example Biggins and Kyprianou [3]. Under (1.2), we have $\gamma=0$ in (1.1).

For discussions on the nature of the assumption (1.2), see Jaffuel [9]. Loosely speaking, letting $\underline{m}$ denote the essential infimum of $\min _{|x|=1} V(x)$, then under some mild integrability conditions, a branching random walk can always be made to satisfy (1.2) after a suitable change of scale, if either $\underline{m}=-\infty$, or $\underline{m}>-\infty$ and $\mathbf{E}\left[\sum_{|x|=1} \mathbf{1}_{\{x=\underline{m}\}}\right]<1$.

In addition of (1.2), we assume the following integrability condition: there exists $\delta>0$ such that

$$
\mathbf{E}\left(\sum_{|x|=1} \mathrm{e}^{\delta V(x)}\right)<\infty, \quad \mathbf{E}\left(\sum_{|x|=1} V(x) \mathrm{e}^{-(1+\delta) V(x)}\right)<\infty, \quad \mathbf{E}\left[\left(\sum_{|x|=1} 1\right)^{1+\delta}\right]<\infty .
$$

Theorem 1.1 ([8], [1]) Assume (1.2) and (1.3). On the set of non-extinction, we have

$$
\frac{1}{\log n} \min _{|x|=n} V(x) \rightarrow \frac{3}{2}, \quad \text { in probability. }
$$


The proofs of Theorem 1.1 presented in [8] and [1] are totally different, but both of them are rather technical. [In [1], it is furthermore assumed that the random variables $V(x)$, $|x|=1$, are i.i.d. and that $\sum_{|x|=1} 1$ is a.s. bounded.]

The sole goal of this note is to give a simple and self-contained proof of Theorem 1.1, using only elementary properties of sums of i.i.d. real-valued random variables. We list in the Appendix these elementary properties of sums of i.i.d. real-valued random variables.

Theorem 1.1 is equivalent to saying that for any $\alpha<\frac{3}{2}$ and $\beta>\frac{3}{2}$,

$$
\begin{aligned}
& \mathbf{P}\left\{\min _{|x|=n} V(x) \leq \alpha \log n \mid \text { non-extinction }\right\} \rightarrow 0, \\
& \mathbf{P}\left\{\min _{|x|=n} V(x) \geq \beta \log n \mid \text { non-extinction }\right\} \rightarrow 0, \quad n \rightarrow \infty .
\end{aligned}
$$

We prove (1.5) and (1.6) in Sections 2 and 3, respectively. Our proof of (1.6) is presented under an additional assumption: $\mathbf{E}\left\{\left[\sum_{|x|=1} 1\right]^{2}\right\}<\infty$ and $\mathbf{E}\left\{\left[\sum_{|x|=1} \mathrm{e}^{-(1+\delta) V(x)}\right]^{2}\right\}<\infty$ for some $\delta>0$. It is possible to adapt the proof without using the additional assumption, by means of a truncation argument (see, for example, Lemma 4.5 of Gantert et al. [6]) and at the cost of an extra page, but we think it is more interesting to keep the proof as simple as possible.

Throughout the paper, we use $a_{n} \sim b_{n}(n \rightarrow \infty)$ to denote $\lim _{n \rightarrow \infty} \frac{a_{n}}{b_{n}}=1$; the letter $c$ with subscript denotes a finite and positive constant.

Remark. In order to make our proof truly self-contained, we reprove all known results for branching random walks that are needed in the paper.

\section{Proof of $(\mathbf{1 . 5})$}

For any vertex $x$, let $\llbracket \varnothing, x \rrbracket$ be the unique shortest path relating $x$ to the root $\varnothing$, and $x_{i}$ (for $0 \leq i \leq|x|)$ the vertex on $\llbracket \varnothing, x \rrbracket$ such that $\left|x_{i}\right|=i$.

We assume (1.2). Let $S_{1}, S_{2}-S_{1}, S_{3}-S_{2}, \cdots$ be i.i.d. random variables such that

$$
\mathbf{P}\left\{S_{1} \leq u\right\}=\mathbf{E}\left\{\sum_{|x|=1} \mathrm{e}^{-V(x)} \mathbf{1}_{\{V(x) \leq u\}}\right\}, \quad \forall u \in \mathbb{R} .
$$

In particular, $\mathbf{E}\left(S_{1}\right)=0$. We write $\underline{S}_{n}:=\min _{1 \leq i \leq n} S_{i}$ for $n \geq 1$.

We claim that for any $n \geq 1$ and any measurable function $g: \mathbb{R}^{n} \rightarrow[0, \infty)$,

$$
\mathbf{E}\left\{\sum_{|x|=n} g\left(V\left(x_{1}\right), \cdots, V\left(x_{n}\right)\right)\right\}=\mathbf{E}\left\{\mathrm{e}^{S_{n}} g\left(S_{1}, \cdots, S_{n}\right)\right\} .
$$


This is easily checked 11 by induction (on $n)$ : For $n=1$, (2.2) is nothing else but (2.1). Assume that (2.2) is proved for $n$. Then by conditioning on the first generation of the branching random walk and using the induction hypothesis, we obtain the claimed equality for $n+1$.

Proof of (1.5). Let $K>0$ and $0<a<\frac{3}{2}$. Let

$$
Z_{n}:=\sum_{|x|=n} \mathbf{1}_{\left\{V(x) \leq a \log n, V\left(x_{i}\right) \geq-K, \forall 1 \leq i \leq n\right\}} .
$$

By (2.2), we have,

$$
\mathbf{E}\left(Z_{n}\right)=\mathbf{E}\left\{\mathrm{e}^{S_{n}} \mathbf{1}_{\left\{S_{n} \leq a \log n, S_{i} \geq-K, \forall 1 \leq i \leq n\right\}}\right\} \leq n^{a} \mathbf{P}\left\{S_{n} \leq a \log n, \underline{S}_{n} \geq-K\right\} .
$$

For $n$ such that $a \log n \geq 1$, we have $\mathbf{P}\left\{S_{n} \leq a \log n, \underline{S}_{n} \geq-K\right\} \leq c_{1} \frac{(\log n)^{2}}{n^{3 / 2}}$ (Lemma A.1). Since $a<\frac{3}{2}$, it follows that $\lim _{n \rightarrow \infty} \mathbf{E}\left(Z_{n}\right)=0$.

Under the assumption $\mathbf{E}\left[\sum_{|x|=1} \mathrm{e}^{-V(x)}\right]=1,\left(\sum_{|x|=n} \mathrm{e}^{-V(x)}, n \geq 0\right)$ is a (non-negative) martingale with respect to its natural filtration; so it converges almost surely. In particular, $\sup _{n} \sum_{|x|=n} \mathrm{e}^{-V(x)}<\infty$ a.s. A fortiori, $\inf _{n} \min _{|x|=n} V(x)>-\infty$ a.s. 2

Since we have already proved that $Z_{n} \rightarrow 0$ in probability, this implies (1.5).

\section{Proof of $(\underline{1.6})$}

The proof of (1.6) also relies on the study of the associated random walk $\left(S_{i}\right)$. It is technically slightly more involved, because we use a second-moment argument this time. We start with the observation 3 that, under assumption (1.3), there exists a constant $c_{2}<\infty$ such that, on the set of non-extinction,

$$
\limsup _{n \rightarrow \infty} \frac{1}{n} \max _{|x|=n} V(x) \leq c_{2}, \quad \text { a.s. }
$$

Indeed, by (1.3), there exists $\delta>0$ such that $c_{3}:=\mathbf{E}\left[\sum_{|x|=1} \mathrm{e}^{\delta V(x)}\right]<\infty$. Let $a>\frac{\log c_{3}}{\delta}$, and let $Y_{n}:=\sum_{|x|=n} \mathbf{1}_{\{V(x) \geq a n\}}$. Clearly, $Y_{n} \leq \mathrm{e}^{-a n \delta} \sum_{|x|=n} \mathrm{e}^{\delta V(x)}$. By Chebyshev's inequality, $\mathbf{P}\left(Y_{n}>0\right) \leq \mathbf{E}\left(Y_{n}\right) \leq \mathrm{e}^{-a n \delta} \mathbf{E}\left[\sum_{|x|=n} \mathrm{e}^{\delta V(x)}\right]=\mathrm{e}^{-a n \delta}\left(c_{3}\right)^{n}$, which is summable in $n$ because

\footnotetext{
${ }^{1}$ See the last Remark in the Introduction. There is a deep explanation to the presence of the new random walk $\left(S_{i}\right)$ using the size-biased branching random walk of Lyons, Pemantle and Peres 14 and Lyons 13 . This idea has been used by many authors in various forms, going back at least to Kahane and Peyrière [10].

${ }^{2}$ See the last Remark in the Introduction. In fact, assumption (1.2) ensures that $\min _{|x|=n} V(x) \rightarrow \infty$ a.s. on the set of non-extinction; see Lyons [13.

${ }^{3}$ See the last Remark in the Introduction. It is, obviously, an immediate consequence of (1.1).
} 
$c_{3}<\mathrm{e}^{a \delta}$. By the Borel-Cantelli lemma, $\lim _{n \rightarrow \infty} Y_{n}=0$ a.s. on the set of non-extinction, yielding (3.1).

Proof of (1.6). Let $C>0$ be the constant in Lemma A.3. Let

$$
I_{k}=I_{k}(n):= \begin{cases}{[0, \infty)} & \text { if } 1 \leq k \leq n \\ {\left[\frac{3}{2} \log n, \infty\right)} & \text { if } n<k<2 n \\ {\left[\frac{3}{2} \log n, \frac{3}{2} \log n+2 C\right]} & \text { if } k=2 n\end{cases}
$$

Consider the random variable

$$
\begin{aligned}
Z_{n} & :=\sum_{|x|=2 n} \mathbf{1}_{\left\{V(x) \in\left[\frac{3}{2} \log n, \frac{3}{2} \log n+2 C\right], \min _{1 \leq i \leq n} V\left(x_{i}\right) \geq 0, \min _{n<j \leq 2 n} V\left(x_{j}\right) \geq \frac{3}{2} \log n\right\}} \\
& =\sum_{|x|=2 n} \mathbf{1}_{\left\{V\left(x_{k}\right) \in I_{k}, \forall 1 \leq k \leq 2 n\right\}} .
\end{aligned}
$$

Applying (2.2) gives that

$$
\mathbf{E}\left(Z_{n}\right)=\mathbf{E}\left\{\mathrm{e}^{S_{2 n}} \mathbf{1}_{\left\{S_{k} \in I_{k}, \forall 1 \leq k \leq 2 n\right\}}\right\} \geq n^{3 / 2} \mathbf{P}\left\{S_{k} \in I_{k}, \forall 1 \leq k \leq 2 n\right\} .
$$

By Lemma A.3, this yields $\mathbf{E}\left(Z_{n}\right) \geq c_{4}$ for some constant $c_{4}>0$.

We now estimate the second moment. By definition,

$$
\begin{aligned}
\mathbf{E}\left(Z_{n}^{2}\right) & =\mathbf{E}\left\{\sum_{|x|=2 n} \sum_{|y|=2 n} \mathbf{1}_{\left\{V\left(x_{k}\right), V\left(y_{k}\right) \in I_{k}, \forall 1 \leq k \leq 2 n\right\}}\right\} \\
& =\mathbf{E}\left(Z_{n}\right)+\mathbf{E}\left\{\sum_{j=0}^{2 n-1} \sum_{|z|=j} \mathbf{1}_{\left\{V\left(z_{i}\right) \in I_{i}, \forall 1 \leq i \leq j\right\}} \sum_{\left(x_{j+1}, y_{j+1}\right)} \sum_{(x, y)} \mathbf{1}_{\left\{V\left(x_{k}\right), V\left(y_{k}\right) \in I_{k}, \forall j<k \leq 2 n\right\}}\right\} \\
& =\mathbf{E}\left(Z_{n}\right)+\Lambda_{n},
\end{aligned}
$$

where, the double sum $\sum_{\left(x_{j+1}, y_{j+1}\right)}$ is over pairs $\left(x_{j+1}, y_{j^{+1}}\right)$ of distinct children of $z$, whereas $\sum_{(x, y)}$ is over pairs $(x, y)$ with $|x|=|y|=2 n$ such that $x \geq x_{j+1}$ and $y \geq y_{j+1}$.

Applying the Markov property at generation $j+1$ gives that

$$
\begin{aligned}
\Lambda_{n}=\mathbf{E}\left\{\sum_{j=0}^{2 n-1} \sum_{|z|=j} \mathbf{1}_{\left\{V\left(z_{i}\right) \in I_{i}, \forall 1 \leq i \leq j\right\}} \times\right. & \\
& \left.\times \sum_{\left(x_{j+1}, y_{j+1}\right)} \mathbf{1}_{\left\{V\left(x_{j+1}\right), V\left(y_{j+1}\right) \in I_{j+1}\right\}} f_{j, n}\left(V\left(x_{j+1}\right)\right) f_{j, n}\left(V\left(y_{j+1}\right)\right)\right\},
\end{aligned}
$$

\footnotetext{
${ }^{4}$ By $x \geq y$, we mean either $x=y$, or $y$ is an ancestor of $x$.
} 
where, for any $u \in \mathbb{R}$,

$$
f_{j, n}(u):=\mathbf{E}\left\{\sum_{|x|=2 n-j-1} \mathbf{1}_{\left\{u+V\left(x_{\ell}\right) \in I_{\ell+j+1}, \forall 1 \leq \ell \leq 2 n-j-1\right\}}\right\} .
$$

Applying (2.2), we get:

$$
\begin{aligned}
f_{j, n}(u) & =\mathbf{E}\left\{\mathrm{e}^{S_{2 n-j-1}} \mathbf{1}_{\left\{u+S_{\ell} \in I_{\ell+j+1}, \forall 1 \leq \ell \leq 2 n-j-1\right\}}\right\} \\
& \leq n^{3 / 2} \mathrm{e}^{2 C-u} \mathbf{P}\left\{\underline{S}_{2 n-j-1} \geq-u, \frac{3}{2} \log n-u \leq S_{2 n-j-1} \leq \frac{3}{2} \log n-u+2 C\right\},
\end{aligned}
$$

where $\underline{S}_{k}:=\min _{1 \leq i \leq k} S_{i}$ as before. By (A.1),

$$
f_{j, n}(u) \leq n^{3 / 2} \mathrm{e}^{2 C-u} \mathbf{P}\left\{\frac{3}{2} \log n-u \leq S_{2 n-j-1} \leq \frac{3}{2} \log n-u+2 C\right\} \leq c_{5} \frac{n^{3 / 2} \mathrm{e}^{-u}}{(2 n-j)^{1 / 2}} .
$$

This inequality turns out to be too rough when $j \leq n$, so we do differently in the latter situation. Since $2 n-j-1 \geq n-1$ this time, we apply Lemma A.1 for $u \in I_{j+1}$,

$$
\begin{aligned}
f_{j, n}(u) & \leq n^{3 / 2} \mathrm{e}^{2 C-u} \mathbf{P}\left\{\underline{S}_{2 n-j-1} \geq-u, S_{2 n-j-1} \leq \frac{3}{2} \log n-u+2 C\right\} \\
& \leq c_{6} n^{3 / 2} \mathrm{e}^{-u} \frac{(u+1)(u+\log n)^{2}}{(2 n-j-1)^{3 / 2}} \\
& \leq c_{6} \mathrm{e}^{-u}(u+1)(u+\log n)^{2} \\
& \leq c_{7}(\log n)^{2} \mathrm{e}^{-u}(u+1)^{3}
\end{aligned}
$$

Let us go back to (3.2), to see that

$$
\begin{aligned}
\Lambda_{n} \leq c_{7}^{2}(\log n)^{4} \mathbf{E}\left\{\sum_{j=0}^{n} \sum_{|z|=j} \mathbf{1}_{\left\{V\left(z_{i}\right) \in I_{i}, \forall 1 \leq i \leq j\right\}} \times\right. \\
\left.\times \sum_{\left(x_{j+1}, y_{j+1}\right)} \mathrm{e}^{-V\left(x_{j+1}\right)-V\left(y_{j+1}\right)}\left|V\left(x_{j+1}\right)+1\right|^{3}\left|V\left(y_{j+1}\right)+1\right|^{3}\right\} \\
+c_{5}^{2} n^{3} \mathbf{E}\left\{\sum_{j=n+1}^{2 n-1} \frac{1}{2 n-j} \sum_{|z|=j} \mathbf{1}_{\left\{V\left(z_{i}\right) \in I_{i}, \forall 1 \leq i \leq j\right\}} \sum_{\left(x_{j+1}, y_{j+1}\right)} \mathrm{e}^{-V\left(x_{j+1}\right)-V\left(y_{j+1}\right)}\right\} .
\end{aligned}
$$

We observe that $\sum_{\left(x_{j+1}, y_{j+1}\right)} \mathrm{e}^{-V\left(x_{j+1}\right)-V\left(y_{j+1}\right)}\left|V\left(x_{j+1}\right)+1\right|^{3}\left|V\left(y_{j+1}\right)+1\right|^{3}$ is bounded by $\left[\sum_{x_{j+1}} \mathrm{e}^{-V\left(x_{j+1}\right)}\left|V\left(x_{j+1}\right)+1\right|^{3}\right]^{2}$, whereas $\sum_{\left(x_{j+1}, y_{j+1}\right)} \mathrm{e}^{-V\left(x_{j+1}\right)-V\left(y_{j+1}\right)}$ by $\left[\sum_{x_{j+1}} \mathrm{e}^{-V\left(x_{j+1}\right)}\right]^{2}$. Under the additional assumption that $\mathbf{E}\left\{\left[\sum_{|x|=1} 1\right]^{2}\right\}+\mathbf{E}\left\{\left[\sum_{|x|=1} \mathrm{e}^{-(1+\delta) V(x)}\right]^{2}\right\}<\infty$ for 
some $\delta>0$, we have $\mathbf{E}\left\{\left[\sum_{|x|=1} \mathrm{e}^{-V(x)}|V(x)+b+1|^{3}\right]^{2}\right\} \leq c_{8}(b+1)^{6}$ for some $c_{8}>0$ and all $b \geq 0$. Therefore,

$$
\begin{aligned}
\Lambda_{n} \leq & c_{9}(\log n)^{4} \mathbf{E}\left\{\sum_{j=0}^{n} \sum_{|z|=j} \mathbf{1}_{\left\{V\left(z_{i}\right) \in I_{i}, \forall 1 \leq i \leq j\right\}} \mathrm{e}^{-2 V(z)}(V(z)+1)^{6}\right\} \\
& +c_{10} n^{3} \mathbf{E}\left\{\sum_{j=n+1}^{2 n-1} \frac{1}{2 n-j} \sum_{|z|=j} \mathbf{1}_{\left\{V\left(z_{i}\right) \in I_{i}, \forall 1 \leq i \leq j\right\}} \mathrm{e}^{-2 V(z)}\right\} .
\end{aligned}
$$

Applying (2.2), this leads to:

$$
\Lambda_{n} \leq c_{9}(\log n)^{4} \sum_{j=0}^{n} \mathbf{E}\left\{\mathrm{e}^{-S_{j}}\left(S_{j}+1\right)^{6} \mathbf{1}_{\left\{\underline{S}_{j} \geq 0\right\}}\right\}+c_{10} n^{3} \sum_{j=n+1}^{2 n-1} \frac{1}{2 n-j} \mathbf{E}\left\{\mathrm{e}^{-S_{j}} \mathbf{1}_{\left\{S_{i} \in I_{i}, \forall 1 \leq i \leq j\right\}}\right\} .
$$

It is easy to bound the two expectation expressions on the right-hand side. For the first expectation, we simply use Lemma A.1 to see that $\mathbf{E}\left\{\mathrm{e}^{-S_{j}}\left(S_{j}+1\right)^{6} \mathbf{1}_{\left\{\underline{S}_{j} \geq 0\right\}}\right\} \leq \frac{c_{11}}{(j+1)^{3 / 2}}$ for some $c_{11}>0$ and all $j \geq 0$. For the second, we recall that for $j \in[n+1,2 n-1] \cap \mathbb{Z}, S_{j} \in I_{j}$ means $S_{j} \geq \frac{3}{2} \log n$, so that

$$
\begin{aligned}
\mathbf{E}\left\{\mathrm{e}^{-S_{j}} \mathbf{1}_{\left\{S_{i} \in I_{i}, \forall 1 \leq i \leq j\right\}}\right\} & \leq \mathbf{E}\left\{\mathrm{e}^{-S_{j}} \mathbf{1}_{\left\{\underline{S}_{j-1} \geq 0, \frac{3}{2} \log n \leq S_{j}<3 \log n\right\}}\right\}+\frac{1}{n^{3}} \\
& \leq \frac{1}{n^{3 / 2}} \mathbf{P}\left\{\underline{S}_{j-1} \geq 0, \frac{3}{2} \log n \leq S_{j}<3 \log n\right\}+\frac{1}{n^{3}} \\
& \leq \frac{1}{n^{3 / 2}} c_{19} \frac{9(\log n)^{2}}{j^{3 / 2}}+\frac{1}{n^{3}},
\end{aligned}
$$

the last inequality being a consequence of Lemma A.1. Accordingly,

$$
\Lambda_{n} \leq c_{9} c_{11}(\log n)^{4} \sum_{j=0}^{n} \frac{1}{(j+1)^{3 / 2}}+c_{10} n^{3} \sum_{j=n+1}^{2 n-1} \frac{1}{2 n-j}\left(\frac{9 c_{19}(\log n)^{2}}{n^{3 / 2} j^{3 / 2}}+\frac{1}{n^{3}}\right) \leq c_{12}(\log n)^{4} .
$$

Since $\mathbf{E}\left(Z_{n}^{2}\right)=\mathbf{E}\left(Z_{n}\right)+\Lambda_{n}$, and $\mathbf{E}\left(Z_{n}\right) \geq c_{4}$, this yields $\mathbf{E}\left(Z_{n}^{2}\right) \leq c_{13}(\log n)^{4}\left[\mathbf{E}\left(Z_{n}\right)\right]^{2}$. By the Cauchy-Schwarz inequality, $\mathbf{P}\left\{Z_{n}>0\right\} \geq \frac{\left[\mathbf{E}\left(Z_{n}\right)\right]^{2}}{\mathbf{E}\left(Z_{n}^{2}\right)} \geq \frac{1}{c_{13}(\log n)^{4}}$; hence

$$
\mathbf{P}\left\{\min _{|x|=2 n} V(x) \leq \frac{3}{2} \log n+2 C\right\} \geq \mathbf{P}\left\{Z_{n}>0\right\} \geq \frac{1}{c_{13}(\log n)^{4}}
$$

We obviously can apply the same argument to study $\min _{|x|=2 n-1} V(x)$, to see that for some constants $\widetilde{C}>0$ and $c_{14}>0$, and all $n \geq 2$,

$$
\mathbf{P}\left\{\min _{|x|=n} V(x) \leq \frac{3}{2} \log n+\widetilde{C}\right\} \geq \frac{1}{c_{14}(\log n)^{4}} .
$$


Let5 $\varepsilon>0$ and let $\tau_{n}:=\inf \left\{k: \#\{x:|x|=k\} \geq n^{\varepsilon}\right\}$. For all large $n$,

$$
\begin{aligned}
& \mathbf{P}\left\{\tau_{n}<\infty, \max _{k \in\left[\frac{n}{2}, n\right]} \min _{|x|=k+\tau_{n}} V(x)>\max _{|y|=\tau_{n}} V(y)+\frac{3}{2} \log n+\widetilde{C}\right\} \\
\leq & \sum_{k \in\left[\frac{n}{2}, n\right]} \mathbf{P}\left\{\tau_{n}<\infty, \min _{|x|=k+\tau_{n}} V(x)>\max _{|y|=\tau_{n}} V(y)+\frac{3}{2} \log n+\widetilde{C}\right\} \\
\leq & \sum_{k \in\left[\frac{n}{2}, n\right]}\left[\mathbf{P}\left\{\min _{|x|=k} V(x)>\frac{3}{2} \log n+\widetilde{C}\right\}\right]^{\left\lfloor n^{\varepsilon}\right\rfloor},
\end{aligned}
$$

which, according to (3.3), is summable in $n$. By the Borel-Cantelli lemma, a.s. for all sufficiently large $n$, we have either $\tau_{n}=\infty$, or $\max _{k \in\left[\frac{n}{2}, n\right]} \min _{|x|=k+\tau_{n}} V(x) \leq \max _{|y|=\tau_{n}} V(y)+$ $\frac{3}{2} \log n+\widetilde{C}$. By (3.1), on the system's non-extinction, a.s. for all large $n$, we have either $\tau_{n}=\infty$, or $\max _{k \in\left[\frac{n}{2}, n\right]} \min _{|x|=k+\tau_{n}} V(x) \leq c_{15} \tau_{n}+\frac{3}{2} \log n+\widetilde{C}$.

Recall that the number of particles in each generation forms a super-critical GaltonWatson tree. In particular, on the system's non-extinction, $\frac{\#\{x:|x|=k\}}{m^{k}}$ converges 6 a.s. to a positive random variable when $k \rightarrow \infty$, which implies $\frac{\tau_{n}}{\log n} \rightarrow \frac{\varepsilon}{\log m}$, a.s. $(n \rightarrow \infty)$, and $\max _{k \in\left[\frac{n}{2}, n\right]} \min _{|x|=k+\tau_{n}} V(x) \geq \min _{|x|=n} V(x)$ a.s. for all large $n$. As a consequence, upon the system's survival, we have, a.s. for all large $n$,

$$
\min _{|x|=n} V(x) \leq\left(\frac{3}{2}+\frac{2 \varepsilon c_{15}}{\log m}\right) \log n+\widetilde{C} .
$$

Since $\varepsilon>0$ can be as small as possible, this yields: on the set of non-extinction,

$$
\limsup _{n \rightarrow \infty} \frac{1}{\log n} \min _{|x|=n} V(x) \leq \frac{3}{2}, \quad \text { a.s. }
$$

A fortiori, we obtain (1.6).

\section{A Appendix on sums of i.i.d. random variables}

We list a few elementary properties of one-dimensional random walks needed in this note; they are either known results in the literature, or simple consequences of known results. Let $S_{1}, S_{2}-S_{1}, S_{3}-S_{2}, \cdots$ be i.i.d. real-valued random variables such that $\mathbf{E}\left(S_{1}\right)=0$ and that $0<\mathbf{E}\left(S_{1}^{2}\right)<\infty$. A trivial consequence of Stone's local limit theorem is that there exist constants $c_{16}>0$ and $C_{0}>0$ such that

$$
\sup _{r \in \mathbb{R}} \mathbf{P}\left\{r \leq S_{n} \leq r+h\right\} \leq c_{16} \frac{h}{n^{1 / 2}}, \quad \forall n \geq 1, \forall h \geq C_{0}
$$

\footnotetext{
${ }^{5}$ See the last Remark in the Introduction. From here, the proof is routine, following McDiarmid 15.

${ }^{6}$ Here, $m:=\mathbf{E}\left[\sum_{|x|=1} 1\right] \in(1, \infty)$ is the mean reproduction number in the Galton-Watson process.
} 
We also recall (see Kozlov [12]) two well-known estimates for the tail behaviour of $\underline{S}_{n}:=$ $\min _{1 \leq i \leq n} S_{i}$ : for some constant $c_{17}>0$,

$$
\begin{aligned}
\mathbf{P}\left\{\underline{S}_{n} \geq 0\right\} & \sim \frac{c_{17}}{n^{1 / 2}}, \quad n \rightarrow \infty, \\
\limsup _{n \rightarrow \infty} n^{1 / 2} \sup _{u \geq 0} \frac{1}{u+1} \mathbf{P}\left\{\underline{S}_{n} \geq-u\right\} & <\infty .
\end{aligned}
$$

Lemma A.1 Let $C_{0}>0$ be the constant in A.1). There exists $c_{18}>0$ such that for $a \geq 0$, $b \geq-a$ and $n \geq 1$,

$$
\mathbf{P}\left\{b \leq S_{n} \leq b+C_{0}, \underline{S}_{n} \geq-a\right\} \leq c_{18} \frac{\left[(a+1) \wedge n^{1 / 2}\right]\left[(b+a+1) \wedge n^{1 / 2}\right]}{n^{3 / 2}}
$$

where $x \wedge y:=\min \{x, y\}$. In particular, there exists $c_{19}>0$ such that for $a \geq 0, b \geq-a$ and $n \geq 1$,

$$
\mathbf{P}\left\{S_{n} \leq b, \underline{S}_{n} \geq-a\right\} \leq c_{19} \frac{\left[(a+1) \wedge n^{1 / 2}\right]\left[(b+a+1)^{2} \wedge n\right]}{n^{3 / 2}}
$$

Proof. We only need to prove the first inequality.

There is nothing to prove if $n \leq 99$; so let us assume that $n \geq 100$. We present the proof only for the case that $n$ is a multiple of 3 ; say $n=3 k$. A similar argument applies if $n=3 k+1$ or if $n=3 k+2$.

By the Markov property at time $k$, we have

$$
\begin{aligned}
& \mathbf{P}\left\{b \leq S_{3 k} \leq b+C_{0}, \underline{S}_{3 k} \geq-a\right\} \\
\leq & \mathbf{P}\left\{\underline{S}_{k} \geq-a\right\} \sup _{x \geq-a} \mathbf{P}\left\{b-x \leq S_{2 k} \leq b-x+C_{0}, \underline{S}_{2 k} \geq-a-x\right\} .
\end{aligned}
$$

By $(\underline{\mathrm{A} .3}), \mathbf{P}\left\{\underline{S}_{k} \geq-a\right\} \leq c_{20} \frac{(a+1) \wedge k^{1 / 2}}{k^{1 / 2}}$. It remains to check that

$$
\sup _{x \geq-a} \mathbf{P}\left\{b-x \leq S_{2 k} \leq b-x+C_{0}, \underline{S}_{2 k} \geq-a-x\right\} \leq c_{21} \frac{(b+a+1) \wedge k^{1 / 2}}{k}
$$

Let $\widetilde{S}_{j}:=S_{2 k-j}-S_{2 k}$. Then $\mathbf{P}\left\{b-x \leq S_{2 k} \leq b-x+C_{0}, \underline{S}_{2 k} \geq-a-x\right\} \leq \mathbf{P}\left\{-b+x-C_{0} \leq\right.$ $\left.\widetilde{S}_{2 k} \leq-b+x, \min _{1 \leq i \leq 2 k} \widetilde{S}_{i} \geq-a-b-C_{0}\right\}$. By the Markov property, this leads to: for $x \geq-a$,

$$
\begin{aligned}
& \mathbf{P}\left\{b-x \leq S_{2 k} \leq b-x+C_{0}, \underline{S}_{2 k} \geq-a-x\right\} \\
\leq & \mathbf{P}\left\{\min _{1 \leq i \leq k} \widetilde{S}_{i} \geq-a-b-C_{0}\right\} \sup _{y \in \mathbb{R}} \mathbf{P}\left\{-b+x-C_{0}-y \leq \widetilde{S}_{k} \leq-b+x-y\right\} .
\end{aligned}
$$


The first probability expression on the right-hand side is bounded by a constant multiple of $\frac{(b+a+1) \wedge k^{1 / 2}}{k^{1 / 2}}$ (by (A.3) $)$, whereas the second probability expression bounded by a constant multiple of $k^{-1 / 2}$ (by (A.1)). Lemma A.1 is proved.7

Lemma A.2 There exists a constant $C>0$ such that for any $0<a \leq b<\infty$,

$$
\liminf _{n \rightarrow \infty} n^{1 / 2} \inf _{u \in\left[a n^{1 / 2}, b n^{1 / 2}\right]} \mathbf{P}\left\{u \leq S_{n}<u+C \mid \underline{S}_{n} \geq 0\right\}>0 .
$$

Proof. Follows immediately from a conditional local limit theorem (Caravenna [5]): if the distribution of $S_{1}$ is non-lattice (i.e., not supported in any $a+b \mathbb{Z}$, with $a \in \mathbb{R}$ and $b>0$ ), then 8 for any $h>0, \mathbf{P}\left\{r \leq S_{n} \leq r+h \mid \underline{S}_{n} \geq 0\right\}=\frac{h r^{+}}{n \mathbf{E}\left(S_{1}^{2}\right)} \exp \left(-\frac{r^{2}}{2 n \mathbf{E}\left(S_{1}^{2}\right)}\right)+o\left(\frac{1}{n^{1 / 2}}\right), n \rightarrow \infty$, uniformly in $r \in \mathbb{R}$; if the distribution of $S_{1}$ is lattice, and is supported in $a+b \mathbb{Z}$ with $b>0$ being the largest such value (called the "span" in the literature), then $\mathbf{P}\left\{S_{n}=a n+b \ell \mid \underline{S}_{n} \geq\right.$ $0\}=\frac{b(a n+b \ell)^{+}}{n \mathbf{E}\left(S_{1}^{2}\right)} \exp \left(-\frac{(a n+b \ell)^{2}}{2 \mathbf{E}\left(S_{1}^{2}\right)}\right)+o\left(\frac{1}{n^{1 / 2}}\right), n \rightarrow \infty$, uniformly in $\ell \in \mathbb{Z}$.

Lemma A.3 Let $C>0$ be the constant in Lemma A.2. For any sequence $\left(a_{n}\right)$ of nonnegative numbers such that $\lim _{\sup _{n \rightarrow \infty}} \frac{a_{n}}{n^{1 / 2}}<\infty$, we have

$$
\liminf _{n \rightarrow \infty} n^{3 / 2} \mathbf{P}\left\{\underline{S}_{n} \geq 0, \min _{n<j \leq 2 n} S_{j} \geq a_{n}, a_{n} \leq S_{2 n} \leq a_{n}+2 C\right\}>0 .
$$

Proof. Let $c_{22}>0$ and $n_{0} \geq 1$ be such that $a_{n} \leq c_{22} n^{1 / 2}, \forall n \geq n_{0}$. Let $p_{n}$ denote the probability in (A.4). Writing $\lambda_{k}:=2 c_{22} n^{1 / 2}+k C$ for $k \geq 0$, we have, for $n \geq n_{0}+\left\lceil\left(\frac{C}{c_{22}}\right)^{2}\right\rceil$,

$$
\begin{aligned}
p_{n} \geq \sum_{k=0}^{\left\lfloor n^{1 / 2}\right\rfloor} \mathbf{P}\left\{\underline{S}_{n} \geq 0, \lambda_{k} \leq S_{n}<\lambda_{k+1},\right. \\
\left.\min _{n<j \leq 2 n}\left(S_{j}-S_{n}\right) \geq a_{n}-\lambda_{k}, a_{n}-\lambda_{k} \leq S_{2 n}-S_{n} \leq a_{n}+2 C-\lambda_{k+1}\right\} .
\end{aligned}
$$

Note that $2 C-\lambda_{k+1}=C-\lambda_{k}$. By independence, the probability on the right-hand side is

$$
\mathbf{P}\left\{\underline{S}_{n} \geq 0, \lambda_{k} \leq S_{n}<\lambda_{k+1}\right\} \mathbf{P}\left\{\underline{S}_{n} \geq a_{n}-\lambda_{k}, a_{n}-\lambda_{k} \leq S_{n} \leq a_{n}+C-\lambda_{k}\right\} .
$$

The first probability expression is, by (A.2) and Lemma A.2, greater than $\frac{c_{23}}{n}$ (for large $n$ ), uniformly in $0 \leq k \leq\left\lfloor n^{1 / 2}\right\rfloor$, whereas the second is, by writing $\widehat{S}_{j}:=S_{n-j}-S_{n}$, $\geq \mathbf{P}\left\{\min _{1 \leq j \leq n} \widehat{S}_{j} \geq a_{n}-\lambda_{k}+C,-a_{n}-C+\lambda_{k} \leq \widehat{S}_{n} \leq-a_{n}+\lambda_{k}\right\}$, which is $\geq \mathbf{P}\left\{\min _{1 \leq j \leq n} \widehat{S}_{j} \geq\right.$ $\left.0,-a_{n}-C+\lambda_{k} \leq \widehat{S}_{n} \leq-a_{n}+\lambda_{k}\right\}$, and thus by (A.2) and Lemma A.2 again, greater than $\frac{c_{24}}{n}$ (for large $n$ ), uniformly in $0 \leq k \leq\left\lfloor n^{1 / 2}\right\rfloor$. Consequently, for all sufficiently large $n$, $p_{n} \geq \sum_{k=0}^{\left\lfloor n^{1 / 2}\right\rfloor} \frac{c_{23}}{n} \times \frac{c_{23}}{n}$, proving the lemma.

\footnotetext{
${ }^{7}$ We mention that in the case $a=0$, Lemma A.1 is essentially Lemma 20 of Vatutin and Wachtel [17.

${ }^{8}$ For any $r \in \mathbb{R}, r^{+}:=\max \{r, 0\}$ denotes its positive part.
} 


\section{References}

[1] Addario-Berry, L. and Reed, B. (2009). Minima in branching random walks. Ann. Probab. 37, 1044-1079.

[2] Biggins, J.D. (1976). The first- and last-birth problems for a multitype age-dependent branching process. Adv. Appl. Probab. 8, 446-459.

[3] Biggins, J.D. and Kyprianou, A.E. (2005). Fixed points of the smoothing transform: the boundary case. Electron. J. Probab. 10, Paper no. 17, 609-631.

[4] Bolthausen, E. (1976). On a functional central limit theorem for random walks conditioned to stay positive. Ann. Probab. 4, 480-485.

[5] Caravenna, F. (2005). A local limit theorem for random walks conditioned to stay positive. Probab. Theory Related Fields 133, 508-530.

[6] Gantert, N., Hu, Y. and Shi, Z. (2008+). Asymptotics for the survival probability in a killed branching random walk. arXiv:0811.0262

[7] Hammersley, J.M. (1974). Postulates for subadditive processes. Ann. Probab. 2, 652680.

[8] Hu, Y. and Shi, Z. (2009). Minimal position and critical martingale convergence in branching random walks, and directed polymers on disordered trees. Ann. Probab. 37, $742-789$.

[9] Jaffuel, B. (2009+). The critical barrier for the survival of the branching random walk with absorption. arXiv:0911.2227

[10] Kahane, J.-P. and Peyrière, J. (1976). Sur certaines martingales de Mandelbrot. Adv. Math. 22, 131-145.

[11] Kingman, J.F.C. (1975). The first birth problem for an age-dependent branching process. Ann. Probab. 3, 790-801.

[12] Kozlov, M.V. (1976). The asymptotic behavior of the probability of non-extinction of critical branching processes in a random environment. Theory Probab. Appl. 21, 791804.

[13] Lyons, R. (1997). A simple path to Biggins' martingale convergence for branching random walk. In: Classical and Modern Branching Processes (Eds.: K.B. Athreya and P. Jagers). IMA Volumes in Mathematics and its Applications 84, 217-221. Springer, New York.

[14] Lyons, R., Pemantle, R. and Peres, Y. (1995). Conceptual proofs of $L \log L$ criteria for mean behavior of branching processes. Ann. Probab. 23, 1125-1138.

[15] McDiarmid, C. (1995). Minimal positions in a branching random walk. Ann. Appl. Probab. 5, 128-139. 
[16] Révész, P. (1994). Random Walks of Infinitely Many Particles. World Scientific, Singapore.

[17] Vatutin, V.A. and Wachtel, V. (2009). Local probabilities for random walks conditioned to stay positive. Probab. Theory Related Fields 143 177-217.

Elie Aïdékon

Department of Mathematics and Computer Science Technische Universiteit Eindhoven

P.O. Box 513

5600 MB Eindhoven

The Netherlands

elie.aidekon@gmail.com

\section{Zhan Shi}

Laboratoire de Probabilités UMR 7599

Université Paris VI

4 place Jussieu

F-75252 Paris Cedex 05

France

zhan.shi@upmc.fr 